\title{
Kinematics of a Head-Neck Model Simulating Whiplash
}

Giuseppe Colicchia, Ludwig-Maximilians University, D-80799 Munich, Germany

Dean Zollman, * Kansas State University, Manhattan, KS

Hartmut Wiesner, Ludwig-Maximilians University, D-80799 Munich, Germany

Ahmet Ilhan Sen, Hacettepe University, Ankara, Turkey

$\Delta$

whiplash event is a relative motion between the head and torso that occurs in rear-end large inertia of the head results in a horizontal translation relative to the thorax. This paper describes a simulation of the motion of the head and neck during a rear-end (whiplash) collision. A head-neck model that qualitatively undergoes the same forces acting in whiplash and shows the same behavior is used to analyze the kinematics of both the head and the cervical spine and the resulting neck loads. The rapid acceleration during a whiplash event causes the extension and flexion of the cervical spine, which in turn can cause dislocated vertebrae, torn ligaments, intervertebral disc herniation, and other trauma that appear to be the likely causes of subsequent painful headache or neck pain symptoms. Thus, whiplash provides a connection between the dynamics of the human body and physics. Its treatment can enliven the usual teaching in kinematics, and both theoretical and experimental approaches provide an interesting biological context to teach introductory principles of mechanics.

\section{Head-Neck Complex}

The cervical spine consists of seven vertebrae (Fig. 1). The topmost vertebra supports the skull; the last is above the thoracic vertebra. The intervertebral joints are saddle joints; they consist of two concavities facing one another and set at right angles to one another. The

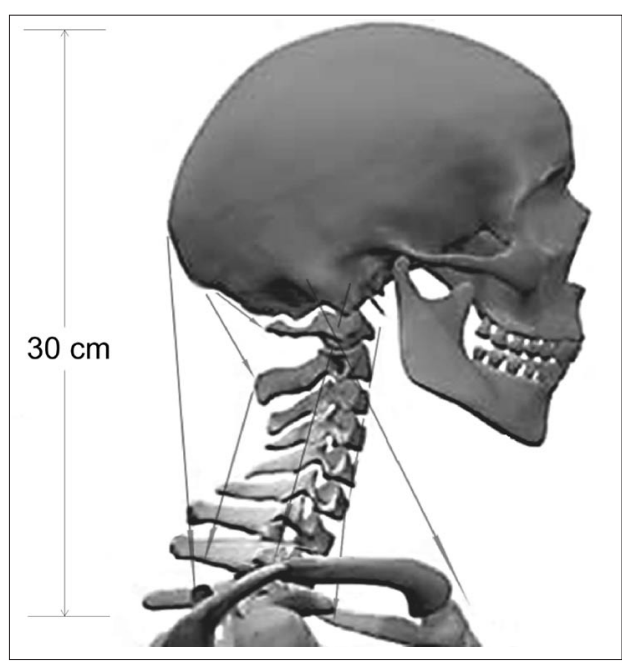

Fig. 1. Simplified head-neck anatomy. The arrows represent the more important muscles.

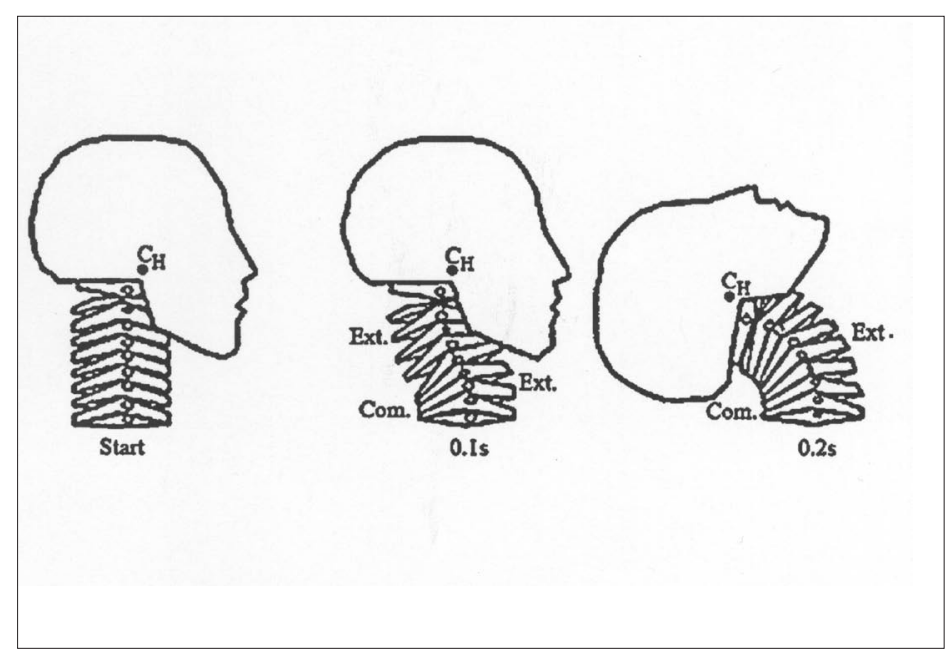

Fig. 2. Schematic drawing of the head-neck motion during whiplash (modified from Mats et al.) ${ }^{1}$ 
vertebrae are separated by intervertebral discs. The disc consists of a nucleus designed to sustain compression loads and an anulus designed to resist tension. The primary function of the discs is to give several degrees of freedom at the spine, sustain loads transmitted from segments above, act as a shock absorber, and distribute forces, eliminating bone-to-bone contact.

The mechanical stability of the cervical spine comes from the intervertebral discs, the ligaments, and the neck muscles. The ligaments (bands) hold the vertebrae together and help to limit the amount of movement of joints. The neck muscles, more than 20 pairs acting with apparent redundancy in various directions, take care of the static and dynamic control of the head and neck. However, the effects of active musculature may be minimal in whiplash, because the muscle contraction occurs late in the dynamic event, when the head-neck is already in extension.

\section{Head-Neck Responses During Whiplash}

In a typical rear-end collision, the vehicle accelerates forward when struck and the torso is pushed forward by the seat. The structural response of the cervical spine is dependent upon the acceleration-time pulse applied to the thoracic spine and interaction of the head and spinal components. During the initial phases of the impact, it is obvious that the lower cervical vertebrae move horizontally faster than the upper ones (Fig. 2). The shear force is transmitted from the lower cervical vertebrae to the upper ones through soft tissues between adjacent vertebrae one level at a time. This shearing motion contributes to the initial development of an S-shape curvature of the neck (the upper cervical spine undergoes flexion while the lower part undergoes extension), which progresses to a C-shape curvature. At the end of the loading phase, the entire head-neck complex is under the extension mode with a single curvature. This implies the stretching of the anterior and compression of the posterior parts of the cervical spine. ${ }^{2,3}$

The forward movement of the trunk displaces the neck forward of the line of gravity of the head (the head mass is about $5 \mathrm{~kg}$ [ $6 \%$ of body mass] with center of mass $C_{\mathrm{H}}$ located a few centimeters above the topmost vertebra). As a result (by $120 \mathrm{~ms}$ ) the center of

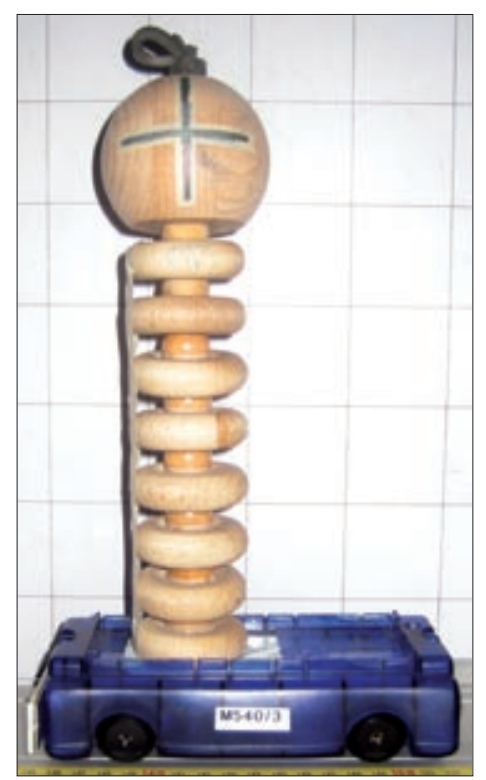

Fig. 3. The head-neck model on a dynamics cart.

gravity of the head starts to drop and causes the head to rotate backward.

Head restraints were introduced in the 1960s as a countermeasure to limit relative motion between the head and thorax. However, the effectiveness of these devices in reducing automotive injury has been limited. Often they are not positioned correctly to constrain head-neck motions within the physiologic range. Therefore, to prevent whiplash injuries, car head retraints must be correctly positioned behind and close to the head. Head restraints, which decelerate simultaneously both the cranium and the cervical spine immediately following rear impact acceleration, have demonstrated some success in reducing whiplash injuries. $^{4,5}$

\section{Experimental Setup}

Whiplash in rear-end car collisions was simulated with a head-neck model on a dynamics cart. The headneck model consists of a column of seven wood rings. The topmost supports a ball that represents the head; the last is above a ring, rigidly attached, that represents the upper thoracic vertebra. The rings are separated by balls, which simulate the cervical intervertebrate joints and give at the rings several degrees of freedom (Fig. 3). An elastic band through wood rings and balls stabilizes the model and simulates all acting forces to hold the head and cervical spine. A soft adhesive tape 


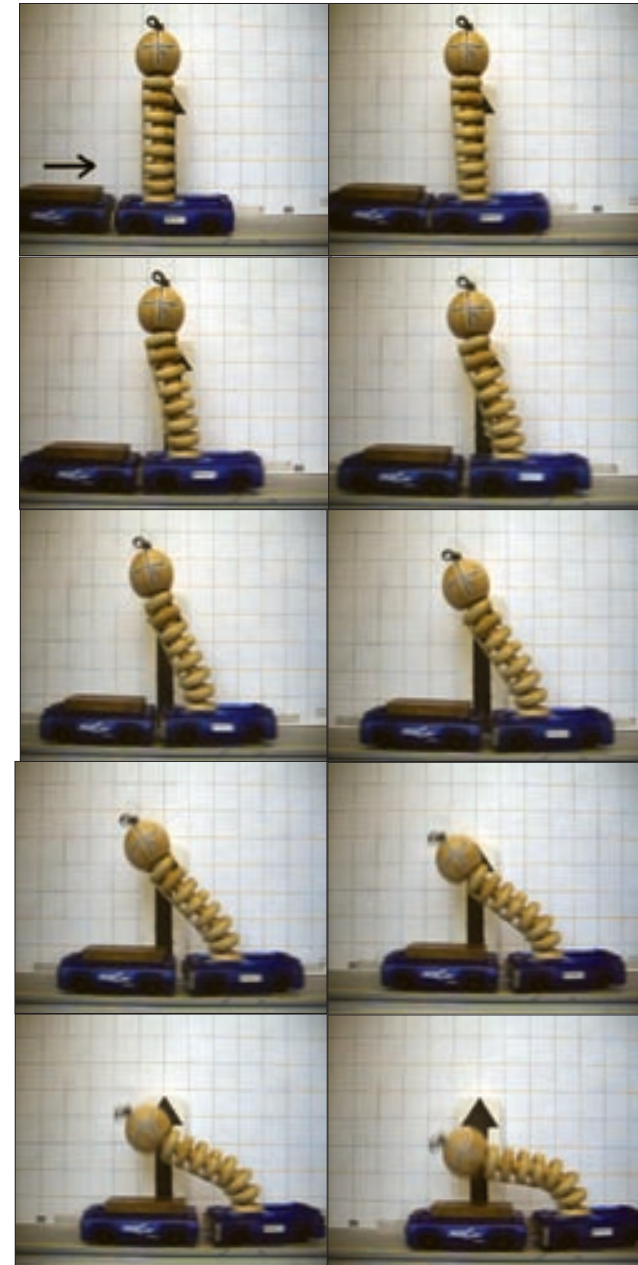

Fig. 4. Motion sequence of the headneck model during a whiplash simulation.

on the back side holds the wood rings in start position, without influencing the kinematics of the model.

Using a usual video camera or digital still camera in video modus (60 frames per second) the pattern of motion of the head and neck model were recorded. From start to full extension, the camera produces about 10 images for each event. When printed and converted to a static view, each image could be compared to any other in the series in order to reconstruct geometrically the pattern of motion.

\section{Qualitative Analysis}

The sequencing of the model's motion during whiplash is visualized in Fig. 4. The model exhibits similar kinematics as that in the cervical spine and so provides a picture of what happens to the neck and head during whiplash. It can be seen that the whiplash mechanism consists of translation/extension of the

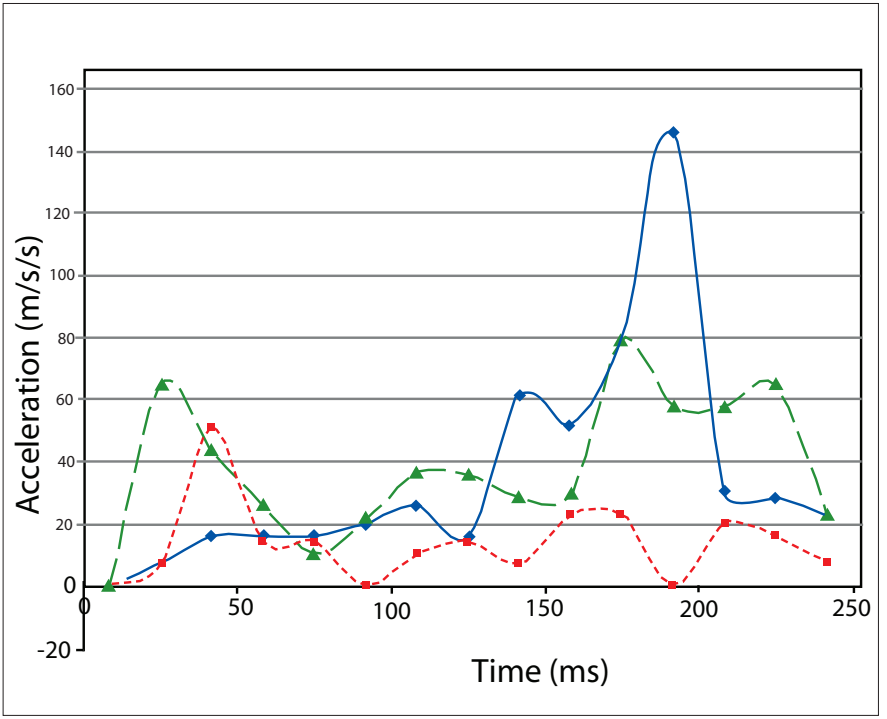

Fig. 5. Acceleration of the head (solid blue line), middle (short red dashes) and bottom (long green dashes) of the model. The collision occurs at time $=1 \mathrm{~ms}$.

cervical spine.

At the start, the center of mass for each body is located midway between the joint. First the lower rings are forward. The forward movement displaces the neck in front of the line of gravity of the ball. As a result, the center of gravity of the ball starts to drop and causes the ball to rotate backward.

\section{Quantitative Analysis}

The motion shown in the video can be analyzed using a program that extracts distance and time data from the images. In our analysis we used the video data collection portion of Coach $6 .{ }^{6}$ This program can be given a set of locations on the object of interest and will collect distance-time data for each of these locations in each frame of the video.

We selected three locations on the model - the head, a midpoint on the "spine," and the location where the model is attached to the cart. With the distance-time data, we calculated the velocity and acceleration for each of these locations during a simulation shown in Fig. 4. Results for the magnitude of the acceleration are shown in Fig. 5.

These graphs show clearly and quantitatively that Newton's first law plays a major role in whiplash injuries. The location at which the model is attached to the cart has a significant acceleration as soon as the collision occurs. For the other points, the acceleration at impact is relatively small. However, the accelerations 
at other points along the model change rapidly during the short time span of approximately $0.16 \mathrm{~s}$. The result is the $\mathrm{S}$-shape motion of the neck and the large forces on the body during the time of the collision, which were discussed above.

\section{Conclusion}

This relatively simple model demonstrates a complex interaction that occurs during an automobile rear-end collision. By using an inexpensive digital camera, students can collect both qualitative and quantitative data from this model. A discussion can focus on the application of physics to automobile safety and the importance of correctly adjusting the head rest in cars. Thus, they can learn both some physics and an important lesson that could keep them from being injured in a low-speed collision.

\section{References}

1. Y. Mats et al., "Neck injuries in car collisions-A review covering a possible injury mechanism and the development of a new rear-impact dummy," Accident Anal. Prev. 32, 167-175 (March 2000).

2. M. Panjabi et al., "Cervical spine curvature during simulated whiplash," Clin. Biomech. 19, 1-9 (Jan. 2004).

3. M. Panjabi et al., "Mechanism of whiplash injury," Clin. Biomech. 13, 239-249 (June-July 1998).

4. B. Stemper et al., "Effect of head restraint backseat on head-neck kinematics in whiplash," Accident Anal. Prev. 38, 317-323 (March 2006).

5. A. Young et al., "Assessing automobile head restraint positioning in Portland, Oregon," Inj. Prev. 11, 97-101 (April 2005); http://ip.bmjjournals.com/.

6. Coach 6 Studio MV (Centre for Microcomputer Applications, Amsterdam, 2006); http://www.cma.science.uva.nl/.

* Supported by the National Science Foundation under grant DUE 04-27645

PACS codes: 45.00.00, 80.00.00

Giuseppe Colicchia is a visiting scientist at LMU-University Munich, Germany; pino@Irz.uni-muenchen.de

Ahmet Ilhan Sen is an associate professor of physics at Hacettepe University, Turkey; ailhan @ hacettepe.edu.tr

Hartmut Wiesner is a professor of physics education at LMU - University Munich, Germany; hartmut.wiesner@ physik. uni-muenchen.de

Dean Zollman is University Distinguished Professor of physics at Kansas State University and a guest professor at Ludwig-Maximilians University in Munich; dzollman@ @hys. ksu.edu 